\title{
La trayectoria de inserción laboral de los jóvenes inmigrantes
}

\author{
Concepción Carrasco Carpio ${ }^{1}$ \\ Alberto Riesco Sanz ${ }^{2}$ \\ 1. Facultat de Ciencias Económicas y Empresariales de la Universidad de Alcalá. \\ Área de Sociología \\ concha.carrasco@uah.es \\ 2. Facultad de Ciencias Económicas y Empresariales de la Universidad Complutense \\ de Madrid. Sección Departamental de Sociología III \\ ariesco@ccee.ucm.es
}

Recibido: 30-03-2008

Aceptado: 01-07-2008

\section{Resumen}

Algunas de las particularidades de los mercados de trabajo en España afectan de manera más pronunciada a la población joven. Se trata de aspectos como la temporalidad, la rotación, la siniestralidad, los bajos salarios o las peores condiciones laborales de algunas ramas de actividad. Este panorama genera trayectorias de empleo diferenciadas para los jóvenes en comparación con la población adulta. En este artículo, trataremos de responder algunas cuestiones, por ejemplo: ¿quiénes son considerados jóvenes?; ¿qué proporción de esta población es de origen inmigrante?; ¡este panorama laboral afecta de manera más dramática a los jóvenes que proceden de familias inmigrantes?; es decir, ¿influye el peso de la etnicidad en la construcción de juventudes diferenciadas, así como en sus transiciones profesionales? Para ello, tenemos un doble objetivo: Primero, establecer el marco de incorporación laboral de los jóvenes, para lo cual analizaremos datos de las fuentes secundarias siguientes: Encuesta de población activa, Encuesta de estructura salarial, Encuesta de transición educativo-formativa e inserción laboral (ETEFIL), Informe Juventud en España 2004, padrón continuo de habitantes, registros de altas en las cotizaciones a la Seguridad Social y registro de jóvenes matriculados en enseñanzas no universitarias y universitarias (Ministerio de Educación y Ciencia). Segundo, determinar los elementos que expliquen, en su caso, las trayectorias laborales diferenciales de los jóvenes procedentes de la inmigración, guiados por la hipótesis de que no es la etnicidad en sí misma la variable más importante que opera en dichas trayectorias diferenciales, sino otras dimensiones, tales como las trayectorias formativas, la disponibilidad para el empleo o las características del «estatuto del inmigrante» en las sociedades de llegada. Para ello, utilizaremos tanto las fuentes secundarias como las respuestas a las entrevistas en profundidad realizadas a dichos jóvenes ${ }^{1}$.

Palabras clave: jóvenes inmigrantes; transición profesional; trayectorias laborales; formación.

1. El material cualitativo utilizado para la elaboración de este artículo (cerca de un centenar de entrevistas abiertas efectuadas en diferentes ciudades a jóvenes de entre 16 y 19 años procedentes de familias de padres extranjeros) procede de los proyectos de investigación siguientes: Identidades en construcción. Estudio de los procesos de adaptación de los adolescentes de familias inmigrantes, proyecto financiado por el Plan Nacional I+D, SEJ 2004-2007 del Ministerios de Educación y Ciencia, e Inmigración y Educación. Un desafio para el siglo XXI (INMED21, FBBVA/SOC04), proyecto financiado por la Fundación BBVA 2005-2007. 
Abstract. The job placement careers of young immigrants

Some of the peculiarities of the Spanish labour markets affect young people dramatically especially aspects such as temporary contracts, turn over, occupational injuries, low salaries and very bad labour conditions in some job sectors. This scenario causes different job careers for young people than for the rest of the employed population. In this article we will try to answer some questions: Who should be considered young people? What proportion of the young are immigrants? Does this scenario affect especially and dramatically young people belonging to immigrated families? In other words, how does ethnicity affect the construction of different youths, as well as their professional careers. Therefore we have a double aim: Firts, to establish a frame for the labour incorporation of the young people. In order to achieve that we are going to examine data coming from the following secondary sources: Encuesta de población activa, Encuesta de estructura salarial, Encuesta de transición educativo-formativa e inserción laboral (ETEFIL), Informe Juventud en España 2004, Padrón continuo de habitantes, Altas en las cotizaciones a la Seguridad Social) and Registro de jóvenes matriculados en enseñanzas no universitarias y universitarias. Second, to determine the elements that might explain different careers of young people belonging to immigrated families. We will follow the hypothesis that ethnicity is not the key element responsible for the youth's differentiation but other dimensions such as learning processes, availability of jobs, or the «immigrant pattern» which characterizes the host society, thereby using both secondary sources and young people's answers to in-depth interviews.

Key words: young immigrants; careers; professional transition; learning processes.

\section{Sumario}

1. La juventud: conceptos y perfiles

2. El marco de inserción laboral de los jóvenes trabajadores
3. La transición profesional de los jóvenes procedentes de familias inmigrantes:

el peso de la etnicidad en la consrucción de juventudes diferenciadas

4. Conclusiones

5. Referencias bibliográficas

\section{La juventud: conceptos y perfiles}

El concepto de juventud es difícil de delimitar desde un punto de vista cualitativo, lo cual complica su delimitación cuantitativa ${ }^{2}$. Pese a estas dificultades, acercaremos al lector algunos datos básicos: cuántos jóvenes hay, cuál es la importancia numérica de los jóvenes procedentes de la inmigración y cuáles son sus perfiles educativos.

2. Desde las ciencias sociales, se ha hecho hincapié en la invención de la «juventud» (inmigrante o no) como sujeto político, social y sociológico, subrayando su carácter heterogéneo y plural, ante lo cual podríamos llegar a preguntarnos si existe eso a lo que llamamos «juventud». Esta problematización del concepto de juventud puede verse, por ejemplo, en Bourdieu (1984), Martín Criado (1998) o, más recientemente, García Borrego (2003). 
Si, tal y como hace el Informe Juventud en España 2004, entendemos por jóvenes a las personas cuya edad está comprendida entre los 16 y los 29 años (López Blasco et al., 2005), el universo en el que centraríamos nuestra atención estaría formado por 8.450 .195 jóvenes, según datos provisionales del censo municipal a 1 de enero de 2008 , lo que supone un 18,3 por ciento de la población empadronada en esa fecha, dato bastante inferior al 30 por ciento que representan los jóvenes inmigrantes (1.562.566 en términos absolutos) entre los inmigrantes totales empadronados (5.268.762). Estos inmigrantes jóvenes proceden, principalmente y en orden de importancia numérica, de Rumanía, Marruecos, Ecuador, Bolivia y Colombia.

Por otra parte, el Observatorio Permanente de la Inmigración (OPI) (con datos proporcionados por el Ministerio del Interior y por el Ministerio de Trabajo e Inmigración) estima que un 27,7 por ciento de la población extranjera con tarjeta o autorización de residencia estaba formado por jóvenes de entre 15 y 29 años (897.609).

En atención a estas primeras pinceladas numéricas, hemos de resaltar, en primer lugar, la mayor juventud relativa de la población inmigrante; en segundo lugar, el mayor peso de los jóvenes inmigrantes entre la población activa (un 34,6 por ciento sobre la población potencialmente activa extranjera) y, por último, las claras consecuencias de rejuvenecimiento en la estructura de población de la pirámide demográfica española.

Centrando la atención en la dimensión académica de estos jóvenes, hemos de reconocer que la educación es una de las variables más importantes a tener en cuenta en las trayectorias formativas y laborales de los jóvenes. Si atendemos a los datos sobre jóvenes matriculados tanto en enseñanzas no universitarias como universitarias en el curso 2007-2008 (Ministerio de Educación y Ciencia), la primera conclusión que obtenemos es que los jóvenes «inmigrantes» ${ }^{3}$ son todavía escasos en las etapas no obligatorias de enseñanza (en enseñanzas no universitarias la proporción es del 9,4 por ciento) y, en segundo lugar, se observa una ligera sobrerrepresentación (véase la tabla 1$)^{4}$ de estos alumnos en formación profesional.

La proporción de jóvenes de procedencia inmigrante que optan por la formación profesional se ha ido incrementando desde comienzos de este siglo. Dicha tendencia ha de ser valorada como significativa para las transiciones profesionales de estos jóvenes, en especial cuando conocemos, por la Encuesta de transición educativo-formativa e inserción laboral (ETEFIL), cual ha sido la experiencia de las transiciones al mundo del trabajo de los jóvenes que estudiaron formación profesional en años anteriores y que se resumen en los siguientes datos: el 67,4 por ciento de los titulados en ciclos formativos de grado

3. En este caso, inmigrante es sinónimo de extranjero, puesto que las estadísticas del MEC se refieren a nacionalidad no española.

4. Para una aproximación más detallada a las características y a la composición del alumnado de origen extranjero en España (incluidas sus pautas de inserción laboral), véase Cachón (2003b) y Colectivo Ioé (2002). 
Tabla 1. Jóvenes totales e inmigrantes matriculados en el curso 2007-2008 según nivel

\begin{tabular}{lccc}
\hline & $\begin{array}{l}\text { Jóvenes } \\
\text { totales }\end{array}$ & $\begin{array}{l}\text { Jóvenes } \\
\text { inmigrantes }\end{array}$ & $\begin{array}{l}\text { \% jóvenes } \\
\text { inmigrantes } \\
\text { sobre el total }\end{array}$ \\
\hline Bachillerato & 619.939 & 27.181 & 4,4 \\
Formación profesional & 510.907 & 34.616 & 6,8 \\
Enseñanzas de régimen especial & 716.177 & 18.397 & 2,6 \\
Educación universitaria & 1.389 .394 & 27.614 & 2 \\
\hline
\end{tabular}

Fuente: Ministerio de Educación y Ciencia.

medio en 2000-2001 trabajaba seis meses después de terminar. Dicho porcentaje se incrementó hasta un 82,3 por ciento al finalizar el período 20012005, momento en el que un 9,1 por ciento buscaba empleo y un 5,4 por ciento continuaba estudiando. En los ciclos de grado superior, trabajaba el 58,7 por ciento seis meses después de terminar. De ello destaca también que un 23,3 por ciento había elegido seguir estudiando; al finalizar el período, trabajaba un 75,8 por ciento, continuaba estudiando un 12,4 por ciento y buscaba empleo un 8,7 por ciento.

\section{El marco de inserción laboral de los jóvenes trabajadores}

La población joven que reside en España, independientemente de su procedencia y que ha tenido o espera tener contacto con los mercados laborales españoles, puede comprobar fácilmente cuáles han sido, o sospecha que serán, las restricciones fundamentales para una inserción satisfactoria en dichos mercados laborales. El panorama no es muy favorable a tal fin ${ }^{5}$.

Los mercados laborales españoles adolecen de estabilidad laboral, en especial aquellos puestos de trabajo que se crean en los denominados «mercados secundarios» (siguiendo la teoría de la dualidad de Piore, 1983), sectores que estaban creando unos 250.000 puestos de trabajo al año (Encuesta de población activa, segundo trimestre $)^{6}$. Esa falta de estabilidad se pone de manifies-

5. En este artículo, no abordaremos los efectos de la crisis económica que comienza a manifestarse a partir del tercer trimestre de 2007 y que habrán de valorarse en un horizonte temporal más amplio. Es por ello que los datos que utilizaremos tendrán como referencia el año 2007.

6. Conviene, no obstante, señalar que la diferenciación entre mercados de trabajo primarios y secundarios (útil, por ejemplo, de cara a subrayar el carácter segmentado y plural de los mercados de trabajo) no debe hacernos olvidar que, si consideramos trayectorias temporales más dilatadas, idénticos puestos de trabajo, empresas y trabajadores pueden pasar (y pasan) de un tipo de mercado laboral a otro. Igualmente, resulta cada vez más común la coexistencia de empleos "característicos» del mercado de trabajo primario y secundario dentro de la misma rama de actividad, de un mismo proceso productivo (en el que participen distintas empresas pertenecientes o no a distintas ramas y sectores), e incluso dentro de la misma empresa, todo lo cual vuelve en ocasiones complicado establecer hasta dónde llega un tipo de mercado de trabajo y otro. 
to en aspectos como la temporalidad, la rotación, la siniestralidad laboral, los bajos salarios y las peores condiciones laborales de algunas ramas de actividad, entre otros aspectos. Todos ellos son motivo de preocupación para los jóvenes, debido, fundamentalmente, a que algunas de las características estructurales de los mercados de trabajo en España les afectan de manera más profunda.

Analicemos, por ejemplo, las tasas de temporalidad y los salarios. Comencemos con las tasas de temporalidad de los jóvenes asalariados de entre 16 y 29 años, las cuales alcanzan el 51,7 por ciento (con diferencias según tramos de edad que van desde el 80 por ciento para los más jóvenes hasta el 40 para el tramo de edad entre 25 y 29 años) en comparación con la media de la población asalariada española, que se aproxima al 30 por ciento. Esto, por una parte, es lógico, dado que han pasado menos tiempo en el mercado de trabajo y no han podido acumular ni el tiempo ni la experiencia suficientes para o bien convertir sus contratos temporales en indefinidos, o bien para que les realicen un contrato indefinido desde el principio. Esto también ocurre entre la población trabajadora nacida en el extranjero, especialmente con los recién llegados a España, aunque, en este caso, las tasas de temporalidad tan sólo bajan hasta el 62 por ciento para el tramo de 25 a 29 años entre la población inmigrante, en comparación con el 40 por ciento para la población española de ese mismo tramo de edad.

Los datos sobre salarios también muestran evidencias diferenciadoras. Según la Encuesta de estructura salarial, la ganancia media de los jóvenes menores de 20 años es un 48 por ciento menor que la ganancia anual media total (19.680,88 euros). A medida que se avanza en edad, la brecha salarial se va reduciendo, aunque en el tramo de 20 a 29 años la diferencia todavía es de un 25 por ciento. En cuanto a la población inmigrante (en concreto, la procedente de Europa no comunitaria, Sudamérica, África y Asia), las diferencias en las ganancias con respecto a la media en el tramo de edad entre los 20 y los 29 años son elevadas (un 14 por ciento menos).

Concentrándonos en las "malas noticias», las tasas de paro de la población joven son más elevadas que las del resto de población. En parte, ello se explica por las altas tasas de actividad (en especial, a partir de los 20 años de edad) no correspondidas por sus tasas de ocupación. Por otro lado, la falta de experiencia laboral, la escasez de cualificación a edades tempranas, las ramas en las que encuentran empleo o el tiempo de residencia (para los jóvenes inmigrados) son todos ellos factores explicativos de estas elevadas tasas de paro. Las "peor paradas» son las mujeres jóvenes situadas en el tramo de edad comprendido entre los 16 y los 19 años (sus tasas de paro alcanzaban, para el segundo trimestre de 2007, el 37,7 por ciento). Adicionalmente, se constata que las tasas de actividad de los jóvenes inmigrantes son más altas que las de los jóvenes españoles, pero sólo en los dos primeros tramos, hasta los 24 años, puesto que después se invierte la tendencia. La tabla número 2 muestra un resumen de las tasas de actividad, ocupación y paro de los más jóvenes por procedencia. 
Tabla 2. Tasas de actividad, ocupación y paro de la población entre 16 y 24 años por procedencia (en \%)

\begin{tabular}{lccccccc}
\hline & \multicolumn{3}{c}{ Españoles } & & \multicolumn{3}{c}{ Inmigrantes } \\
\cline { 2 - 3 } \cline { 6 - 8 } & $\mathbf{1 6 - 1 9}$ años & $\mathbf{2 0 - 2 4}$ & $\mathbf{2 5 - 2 9}$ & & $\mathbf{1 6 - 1 9}$ & $\mathbf{2 0 - 2 4}$ & $\mathbf{2 5 - 2 9}$ \\
\hline Tasa de actividad & 28,9 & $\mathbf{6 4 , 2}$ & $\mathbf{8 6 , 4}$ & & 38,8 & 80,5 & $\mathbf{8 3 , 7}$ \\
Tasa de ocupación & 20,6 & 55,2 & 79,3 & & 27,1 & 65,3 & 76,3 \\
Tasa de paro & 28,8 & 14 & 8,2 & & 30,2 & 18,9 & 8,8 \\
\hline
\end{tabular}

Fuente: «Ficheros de microdatos». Encuesta de población activa, segundo trimestre de 2007.

El último dato que aportaremos para completar el marco está referido a las ocupaciones y los sectores en los que se concentran los jóvenes en nuestros mercados laborales. Si analizamos los flujos en las afiliaciones a la Seguridad Social, comprobamos que el 46 por ciento de las altas laborales producidas durante el año 2007 habían sido altas de jóvenes. Desglosando los datos en grupos de cotización (sinónimo de cualificación de los puestos de trabajo ocupados), descubrimos que el 27,3 por ciento de dichas altas se produjeron en el grupo 10, o sea el de "peones», mientras que tan sólo representaba el 2,2 por ciento el grupo 1, es decir, el de «licenciados e ingenieros». Adicionalmente, si acudimos a las cifras de altas iniciales, lógicamente mayoritarias al tratarse de jóvenes, encontramos nuevamente que el 30 por ciento de dichas altas se produjeron en el grupo 10 (peones). Una primera conclusión parece obvia: en las oportunidades de empleo para los jóvenes ofrecidas por el mercado laboral español, tienen un peso muy importante las ramas de actividad consideradas más descualificadas, al menos como vía de entrada inicial en el mundo del trabajo.

¿Cuál era la situación de los trabajadores inmigrantes afiliados a la Seguridad Social? Podemos apreciar, en la tabla 3, las principales diferencias entre unos y otros en las cotizaciones según regímenes. La población joven extranjera está sobrerrepresentada en dos de los regímenes, a saber: el régimen especial agrario y el régimen especial de empleados de hogar. Ambos, en conjunto, repre-

Tabla 3. Trabajadores jóvenes afiliados a la Seguridad Social a 31 de diciembre de 2006 según procedencia y régimen de cotización

\begin{tabular}{lcccr}
\hline & \multicolumn{2}{c}{$\begin{array}{l}\text { Jóvenes afiliados en alta } \\
\text { a la Seguridad Social }\end{array}$} & \multicolumn{2}{l}{$\begin{array}{l}\text { Jóvenes inmigrantes } \\
\text { afiliados en alta a la } \\
\text { Seguridad Social }\end{array}$} \\
\hline Totales & 4.468 .000 & $100 \%$ & 607.587 & $100 \%$ \\
Régimen general & 3.900 .400 & $87 \%$ & 473.135 & $78 \%$ \\
Régimen empleados de hogar & 43.600 & $1 \%$ & 36.183 & $6 \%$ \\
Régimen especial agrario & 151.600 & $3,5 \%$ & 54.613 & $9 \%$ \\
Régimen especial autónomos & 362.300 & $8 \%$ & 42.848 & $7 \%$ \\
\hline
\end{tabular}

Fuente: Anuario de estadísticas del Ministerio de Trabajo e Inmigración 2007. 
sentan el 15 por ciento de las cotizaciones a la Seguridad Social, en cambio, tan sólo representan algo más de un 4,5 por ciento para la población joven en general.

Las diferencias por género se acentúan aún más en el caso de los jóvenes inmigrantes, puesto que son ellas, las mujeres jóvenes extranjeras, las que trabajan en el régimen de empleados de hogar (un 13 por ciento de las mujeres jóvenes cotizaba por este régimen a 31 de diciembre de 2007) y ellos, los varones, los que trabajan en el régimen especial agrario (un 10 por ciento de los varones jóvenes), aunque, como se puede comprobar, en ambos casos el régimen de cotización principal es el régimen general, con presencia muy importante de los varones en el sector de la construcción, el cual, como sabemos, comenzó a perder empleo desde finales de 2007.

En consecuencia, el marco de inserción laboral de los jóvenes está marcado por la temporalidad de sus contratos, por los bajos salarios, por tasas de paro elevadas y por segmentación en ramas descualificadas. Este marco presenta algunas particularidades en el caso de los jóvenes procedentes de la inmigración, donde comprobamos una temporalidad en sus contratos que origina segmentación, es decir, que tiene un carácter estructural más difícil de solucionar con el paso del tiempo, unos salarios aún más bajos, unas tasas de paro mayores y una concentración más elevada en ramas de actividad descualificadas y desreguladas.

\section{La transición profesional de los jóvenes procedentes de familias inmigrantes: el peso de la etnicidad en la construcción de juventudes diferenciadas}

Las investigaciones empíricas de carácter cuantitativo realizadas sobre la transición profesional de los jóvenes son todavía poco numerosas. En este aspecto, destaca la Encuesta de transición educativo-formativa e inserción laboral (ETEFIL) y la

7. Esta encuesta se llevó a cabo durante el año 2005 fruto de un acuerdo entre el Ministerio de Educación y Ciencia, de Trabajo y Asuntos Sociales, del Instituto Nacional de Estadística y el Servicio Público de Empleo Estatal. Se trata de una importante investigación estadística sobre los itinerarios seguidos por los jóvenes (sólo se consideraron las personas que a fecha 31 de diciembre de 2001 no habían cumplido los 25 años) dentro del sistema educativo (ámbito no universitario), así como sobre las transiciones entre el estudio y el trabajo durante el período 2001-2005. La investigación se organizó en siete colectivos independientes con la referencia al curso académico 2000-2001, a saber: 1) los jóvenes que en el año 2001 se graduaron en ESO, 2) los jóvenes que abandonaron los estudios de ESO, 3) aquellos que se titularon en bachillerato, 4) jóvenes que terminaron ciclos formativos de grado medio (CFGM), 5) estudiantes que terminaron ciclos formativos de grado superior (CFGS), 6) los que finalizaron cursos del Plan Nacional de Formación e Inserción Profesional (FIP) y 7) los que concluyeron programas de escuelas taller y casas de oficio. El tamaño de la muestra ha alcanzado, para el período 2001-2005, a 45.000 jóvenes, de los cuales 216 eran de nacionalidad extranjera, un 5 por mil (no obstante, ese era aproximadamente el peso de los estudiantes extranjeros en la ESO durante el curso 2000-2001, de manera que las etapas de infantil, primaria y ESO eran las de mayor concentración de estudiantes extranjeros en ese curso). (Más información en: www.mec.es/mecd/estadisticas/index.html.) 
encuesta realizada a jóvenes inmigrantes sobre su transición profesional en el último informe sobre la juventud (IJE) $)^{8}$. Suele ser más habitual recurrir a técnicas cualitativas de investigación, como los autores hicieron en un trabajo anterior ${ }^{9}$. En este apartado, centraremos la atención en las características de la transición profesional o laboral de los jóvenes, en especial para los procedentes de países distintos a España, comenzando por la vertiente cuantitativa, para finalizar con algunos de los elementos significativos que los jóvenes procedentes de la inmigración señalaron en las entrevistas de carácter cualitativo en el marco de los proyectos de investigación antes mencionados.

El IJE (2005) señala cuatro rasgos diferenciales de los jóvenes inmigrantes respecto a los autóctonos, a saber:

1) Viven exclusivamente de sus ingresos en una mayor proporción (un 38\%) que los españoles (un 23\%), es decir, se produce un proceso de emancipación económica más elevada en los jóvenes inmigrantes (con la salvedad de que ese proceso de emancipación puede haberse realizado precisamente con motivo de la migración desde su país de origen).

2) Entre los inmigrantes, los trabajos esporádicos son una fuente mucho más importante de recursos dentro de quienes tienen recursos propios.

3) Los jóvenes inmigrantes que tienen ingresos de otras personas perciben en mucha menor medida ingresos de sus padres y mucho más de sus parejas.

4) Los ingresos medios netos que perciben los jóvenes inmigrantes que tienen ingresos personales es un 12 por ciento menor que los de los españoles.

Si centramos la atención en la parte de la encuesta ETEFIL que analiza la transición de los jóvenes al mundo laboral, la mirada hay que dirigirla, tal y como avanzamos en el apartado anterior, a los ciclos formativos de grado medio o superior y comprobamos que son los jóvenes (españoles y inmigrantes) que terminan estos ciclos los que consiguen mayores tasas de empleo estable. De las personas que finalizaron ciclos formativos de grado medio en el curso 20002001 , repartidas casi por igual entre hombres (52 por ciento) y mujeres ( 48 por ciento), casi siete de cada diez (67,4 por ciento) ya estaba trabajando a los seis meses de la finalización de sus estudios. Además, recordemos que el 81,3 por ciento de los jóvenes estaba trabajando en el momento de realizar la encuesta (primavera de 2005). Con el paso del tiempo, los que han realizado un ciclo formativo de grado superior consiguen más empleo. En el momento de la entrevista, sólo un 8,7 por ciento buscaba empleo, frente al 9,1 por ciento de

8. Dicho informe se apoya en una encuesta realizada a 5.014 personas con edades comprendidas entre 16 y 29 años, el 6 por ciento de cuyos casos tenía nacionalidad extranjera. No obstante, en el IJE están notablemente sobrerrepresentados los latinoamericanos e infrarrepresentados los africanos. De forma adicional, la distribución por edades de los entrevistados es diferente para los españoles y los extranjeros, donde entre estos últimos tienen mayor peso los mayores de 25 años, de manera que este hecho podría explicar algunas de las diferencias encontradas entre ambos colectivos.

9. Véase el artículo publicado en la Revista de Educación, 345, enero-abril de 2008, p. 183-203. 
los graduados en ciclos formativos de grado medio y porcentajes superiores al 10 por ciento en los demás casos. Los jóvenes de los CFGS consiguen, además, los mejores empleos (el 40,4 por ciento ocupaba puestos de trabajo técnicos y profesionales de apoyo). Estos datos enlazan con la titulación que les han requerido en el empleo actual, tanto a jóvenes españoles como inmigrantes. En ambos casos, la más requerida ha sido la formación profesional de grado medio y superior (ligeramente sobrerrepresentada la titulación de grado medio entre los jóvenes inmigrantes, con un 48,4 por ciento de los casos frente a un 44,5 de los españoles).

Un dato menos optimista se genera cuando lo analizado es el abandono de los estudios. En atención a los datos proporcionados por dicha encuesta sobre abandono de los estudios de la enseñanza secundaria obligatoria (curso 20002001), el abandono es mayor en la población joven extranjera (un 29,9 por ciento frente a un 15,3 por ciento). Pero los datos aportados por la memoria del CES (2006) nos hacen pensar que dichos datos están incluso infravalorados. Con informes de Eurostat y PISA (2000-2003), dicha memoria confirma los altos porcentajes de abandono escolar que sufre España en el año 2005 con respecto a otros países de la Unión Europea (UE-25): el 30,8 por ciento de los jóvenes entre 18 y 24 años no había completado la educación secundaria de la segunda etapa y no seguía ningún tipo de estudio ni formación, en comparación con un 15,2 por ciento de media de la UE-25.

En consecuencia, una proporción más elevada de jóvenes emancipados conllevaría una proporción más elevada de jóvenes disponibles para el empleo, pues su fuente principal de ingresos no será otra que su propio trabajo o el de sus parejas. De esta manera, es lógica la consecuencia que se refleja en un mayor porcentaje de abandono de los estudios de la enseñanza obligatoria.

De manera adicional, la mayor disponibilidad para el empleo hace a esta población proclive a ocupar nichos laborales caracterizados por malas condiciones de trabajo (los "empleos de inmigrantes») presentes en un mercado fuertemente segmentado. Esto quedaría hasta cierto punto reflejado, por ejemplo, en el orden de prioridades señaladas por los entrevistados de la ETEFIL:

a) Continuar los estudios hasta conseguir un título (un 46 por ciento de los españoles frente a un 37 de los inmigrantes).

b) Conseguir un empleo estable (prácticamente en la misma proporción del 27 por ciento para españoles e inmigrantes).

c) Encontrar un empleo adecuado a su cualificación en el caso de los españoles (9,7 por ciento), mientras que, para los inmigrantes, la tercera prioridad reside en simplemente conseguir un empleo (11,6 por ciento).

Profundicemos en las emancipaciones (entendiendo por emancipados aquellos jóvenes que no viven ni con su padre ni con su madre y, por tanto, son ellos mismos las personas de referencia o sus parejas). La tabla número 4 refleja los porcentajes de emancipación, el porcentaje de los jóvenes casados y el de los jóvenes que están estudiando. 
Tabla 4. Jóvenes emancipados, casados y estudiantes, según tramo de edad y procedencia (en \%)

\begin{tabular}{lcccccccc}
\hline & \multicolumn{3}{c}{ Españoles } & & \multicolumn{3}{c}{ Inmigrantes } \\
\cline { 2 - 3 } \cline { 7 - 9 } & $\mathbf{1 6 - 1 9}$ años & $\mathbf{2 0 - 2 4}$ & $\mathbf{2 5 - 2 9}$ & & $\mathbf{1 6 - 1 9}$ & $\mathbf{2 0 - 2 4}$ & $\mathbf{2 5 - 2 9}$ \\
\hline Emancipados & 0,6 & 4,2 & 16,6 & & 3,3 & 17,7 & 44 \\
Casados & 0 & 1 & 8,1 & & 0,7 & 7,9 & 22,9 \\
Estudiando (reglados) & 16,7 & 13,4 & 4,9 & & 9,8 & 3,6 & 1,5 \\
Estudiando (no reglados) & 3,4 & 5 & 6,6 & & 1,9 & 3 & 3,4 \\
\hline
\end{tabular}

Fuente: «Ficheros de microdatos». Encuesta de población activa, segundo trimestre de 2007.

En primer lugar, encontramos, tal y como señalábamos, que un factor clave a destacar en las transiciones profesionales de los jóvenes inmigrantes es precisamente su mayor emancipación: el 65 por ciento de ellos está emancipado en España, frente a un 21,4 por ciento de los jóvenes españoles (es más evidente la diferencia existente en el tramo de edad comprendido entre los $25 \mathrm{y}$ los 29 años, puesto que el 44 por ciento de los inmigrantes se trata de jóvenes emancipados, frente a un escaso 16,6 por ciento de los españoles). Y, relacionado directamente con su grado de emancipación, encontramos el porcentaje de jóvenes inmigrantes casados que representa más de la cuarta parte de los jóvenes inmigrantes (31,5 por ciento). Por tanto, los jóvenes que no proceden de familias inmigrantes tienden a prolongar en mayor medida la dependencia familiar (en casa de sus padres), con lo cual ralentizan su incorporación al mercado de trabajo.

Un segundo dato que aporta claridad a los resultados encontrados por los trabajos empíricos antes mencionados es el porcentaje de jóvenes que están cursando estudios reglados o bien algún tipo de formación no regla$\mathrm{da}^{10}$. La diferencia entre los jóvenes españoles y los inmigrantes es esclarecedora (véase la tabla 6). Mientras que un 50 por ciento de los jóvenes españoles está cursando algún tipo de formación (reglada o no), los jóvenes inmigrantes que estudian no llegan a representar una cuarta parte. El proyecto migratorio de los jóvenes inmigrantes tiene una prioridad: su inserción en el mercado de trabajo. Esta disponibilidad para el empleo se manifiesta (como pudimos comprobar en la tabla 2) en unas mayores tasas de actividad de los jóvenes inmigrantes menores de 24 años en comparación con los jóvenes españoles.

En tercer lugar, es importante constatar que el fracaso escolar, entendido como la no consecución de la certificación de la enseñanza secundaria obligatoria, medido según los datos EPA, estaba en torno al 22 por ciento de los jóvenes inmigrantes, mientras que para los jóvenes españoles se reduce hasta el 9,8 por ciento. Para los jóvenes inmigrantes, la prolongación del

10. La EPA realiza la pregunta sobre si ha cursado estudios reglados o bien algún curso de formación no reglada durante las cuatro últimas semanas. 
tiempo de formación más allá de los períodos de escolarización obligatoria es percibida a menudo como un obstáculo para la realización del proyecto migratorio.

Pero, ¿qué nos dicen ellos, los jóvenes procedentes de la inmigración? Con respecto a las causas del abandono de los estudios de manera más pre$\mathrm{coz}$, muchos de estos jóvenes ponen en evidencia la necesidad de ganarse la vida, de ayudar a sufragar los gastos familiares, de enviar remesas o simplemente por cuestiones de género, dado que, en igualdad de circunstancias, para determinadas mujeres jóvenes, es más difícil seguir los estudios que para los varones.

Mi padre es cocinero y mi madre también es ayudanta de la cocina y entran por la mañana a las diez y salen a las cinco y medio y vuelven a entrar a las ocho y salen ya a las doce o a la una y yo tengo que cuidar a mis hermanas hasta que salen. (E- 7, mujer marroquí, segunda generación)

\section{¿Por qué abandonaste el colegio tan pronto?}

Mis padres no tenían como mandar... No podían mantener unos estudios, éramos tres chavales pequeños y..., ellos necesitaban una ayuda para casa y que no podían con todos los gastos por lo mismo. No podía mantenernos estudiando como él quisiera que estuviésemos. Entonces tuvimos que ponernos a trabajar, por lo menos yo, trabajar de muy jovencita para poder salir adelante... Ayudar en casa. (E-14, mujer latinoamericana, generación 1,5)

Porque teníamos más gastos y porque mi padre trabajaba él solo y para los libros y todo esto, pues... Éramos tres... Y más cuando yo cumplí dieciséis años..., empecé a trabajar. (E-24, mujer marroquí, generación 1,5)

Este abandono de los estudios desde muy jóvenes tiene también relación con su socialización en los países de origen. Sus padres y sus madres comenzaron a trabajar muy jóvenes o bien ellos mismos han tenido experiencias laborales tempranas. La valoración de los estudios es distinta, puesto que se aprende trabajando.

Allí en Colombia, pues si quieres trabajar de mecánico, pues empiezas de pequeño. Buscas trabajo en una..., en cualquier taller... Te vas allí y dices: «Mira, yo quiero trabajar de fontanero» o «Yo quiero trabajar de carpintero». Te ponen aprendiz desde pequeño y aprendes trabajando..., y sin tener que haber estudiado nada. (E- 19, varón latinoamericano, primera generación)

Porque incluso en Colombia ella ya empezó a trabajar desde los 12 años. Siempre ha estado trabajando, siempre. (E3, varón latinoamericano, generación $1,5)$

Primero empecé, digamos, repartiendo pasteles en los bares y cada vez más, digamos, luego sigues un poco arriba, haces un poco esto y esto, luego te enseñan esto y esto y luego bajas ya a la barra, sirves unos cuantos cafés y luego ya cada vez más, vas aprendiendo cada vez más y más. (E-25, varón marroquí, generación 1,5) 
Se descubre una línea divisoria entre la realidad y las esperanzas de futuro. Las expectativas de muchos jóvenes pasan por la combinación de estudios y trabajo, así como por valorar de forma más meritocrática los estudios en comparación con la realidad que viven en casa a través del trabajo de sus padres y sus madres. Veamos dos ejemplos:

Claro, eso sería más adelante... Me gustaría estudiar, hacer algunos cursos. Claro, no aspiraría a trabajar toda la vida en una cafetería de camarera. Aspiraría a estudiar lo que guste, computación, idiomas o cualquier otra cosa y después..., buscaría un trabajo con respecto a lo que estudie. (E-15, mujer latinoamericana, primera generación)

\section{¿Crees que vale la pena el esfuerzo que haces?}

Sí..., porque aquí, si no estudias, te va a tocar fregar..., y..., por lo que pasa mi madre, pues no quiero pasar lo mismo... Prefiero ahora que puedo y tengo la oportunidad, pues la tengo que aprovechar. (E-27, mujer marroquí, segunda generación)

Creo que vale la pena estudiar, porque de eso depende nuestro futuro. (E-32, mujer de Europa del Este, generación 1,5)

Las expectativas de un futuro mejor son las mantenidas por muchos padres y madres de estos jóvenes, que tienen muy claro el papel que desempeñan en su proyecto migratorio las oportunidades de estudios de sus hijos e hijas.

Pues $n a$, me dijo mi madre que..., que íbamos a venir aquí, que quería cambiar el ambiente..., y..., que quería que nosotros estudiáramos una carrera... Y eso, estamos estudiando. (E-9, mujer marroquí, generación 1,5)

El realismo de los jóvenes entrevistados reaparece al reflexionar sobre las posibilidades de emancipación, en las que encuentran las mismas dificultades que los jóvenes españoles.

Yo porque vivo con mis padres. Si quisiese, eh..., con mi sueldo poder independizarme... Los pisos están en trescientos y pico al mes y te quedarían otros trescientos euros para comer, y con trescientos euros no puedes comer, pagarte las salidas, la ropa, los calzados..., todo... Es imposible, pero eso ya no es solamente para los sudamericanos, sino para el resto de... españolitos de aquí. (E-14, mujer latinoamericana, generación 1,5)

Por último, nos gustaría añadir un aspecto más que afecta a muchos jóvenes que habían comenzado los estudios en sus países de origen y que vieron truncado su proyecto de formación, al ser transformado en un proyecto migratorio familiar, por lo que se han visto obligados a realizar una transición laboral más rápida de lo esperado.

En mi país, hice la prueba de la selectividad. Me fue bien, pero, como me vine, todo eso se fue..., al carajo, como dicen... Y aquí perdí un año, pues para pre- 
parar la selectividad de nuevo, pues..., tienes que volver y..., a saber otros temas que no serían los mismos temas que aquí, pues mira, dije: «Venga, hago un módulo superior» y mi expectativa actualmente es estudiar. (E-30, mujer latinoamericana, generación 1,5 )

Se me fue el ánimo por estudiar... Yo allí estaba en la universidad con mi padre. Él estaba allí estudiando derecho..., y yo iba a la universidad con él. Él se iba para sus estudios y yo me iba para los míos y..., mira, pero aquí lamentablemente no me fue bien. (E-48, varón latinoamericano, generación 1,5)

Empecé mi etapa universitaria en Marruecos. Me matriculé en Filología porque era lo que me gustaba... Cuando me vine para Barcelona, estaba en tercero... Cuando llegué a Barcelona, se me había pasado el plazo de matrícula... Aquí sí que con los plazos son muy estrictos, mientras que en Marruecos te ponen un plazo, pero..., entonces tienes que buscarte la vida. Me salió algo para trabajar, que era cuidar un niño. (E-50, mujer africana)

En resumen, el rápido abandono escolar de estos jóvenes, así como la elección de itinerarios formativos más cortos (formación profesional de grado medio, por ejemplo), ponen en evidencia la urgencia de seguir una transición desde los estudios hasta la experiencia laboral, en un movimiento que muchas veces está acompañado por procesos de socialización que se mueven en la misma dirección. En otros casos, la transición al mundo del trabajo se realiza por necesidad económica, con lo cual se pierde poder negociador y se gana disponibilidad para «cualquier» empleo. Es esta disponibilidad para el empleo la que desempeña un papel básico en las transiciones laborales, detrás de la cual las causas se acumulan y se entremezclan: las necesidades económicas familiares; la emancipación; el joven como núcleo del proyecto migratorio familiar, y la formación como expectativa de un futuro mejor, pero un «lujo» en el momento presente.

\section{Conclusiones}

Las conclusiones de este breve ensayo se mueven en un continuo que va desde los aspectos estructurales, es decir, el marco de inserción laboral, hasta las especificidades de los jóvenes inmigrantes en el proceso de inserción laboral.

Desde un punto de vista estructural:

Las características del mercado de trabajo constituyen un marco de referencia para la inserción tanto de jóvenes españoles como de jóvenes inmigrantes (tengamos en cuenta que el concepto de juventud es plural, la nacionalidad es una variable más añadida a la heterogeneidad del concepto). Dichas características consisten en mayores tasas de paro, mayores tasas de temporalidad, menores salarios, mayor número de altas en la Seguridad Social en los grupos de cotización menos cualificados (con lo cual se produce una fuerte desproporción entre la formación adquirida y la cualificación reconocida); además de, como consecuencias derivadas de lo anterior, la concentración en algunas ramas de actividad de peores condiciones de trabajo (al menos en las prime- 
ras fases de inserción laboral) o la siniestralidad laboral. Toda una cadena de preocupaciones juveniles que hacen que el proceso de inserción laboral sea lento y largo.

Por su parte, los aspectos específicos más destacados de los jóvenes inmigrantes pueden dividirse en tres dimensiones: los aspectos sociodemográficos, la dimensión educativo-formativa y, por último, pero no menos importante, las características de sus transiciones al mundo laboral. Analicémoslas algo más en detalle:

Desde el punto de vista de las características sociodemográficas, cabe resaltar que los jóvenes inmigrantes son proporcionalmente más jóvenes, es decir, que están sobrerrepresentados entre los residentes extranjeros con respecto a los jóvenes españoles. O lo que es lo mismo, existe una mayor representación de los jóvenes inmigrantes entre la población potencialmente activa extranjera.

Desde la óptica educativo-formativa, podemos añadir que el proceso académico de los jóvenes inmigrantes suele ser más corto, es decir, abandonan antes los estudios que los jóvenes españoles. Tan sólo la educación primaria y la secundaria obligatoria están más generalizadas entre ellos, pero, a medida que avanza el nivel de estudios, los jóvenes inmigrantes son menos numerosos. Para ellos, la prolongación del tiempo de formación más allá de los períodos de escolarización obligatoria es percibida como un obstáculo para la realización del proyecto migratorio.

Por último, la mayor y más precoz emancipación económica de los jóvenes inmigrantes se convierte en una de las características básicas explicativas de las transiciones al mundo laboral. Su disponibilidad para el empleo es una cuestión de necesidad.

Estas tres dimensiones están lógicamente interrelacionadas. Una mayor proporción de jóvenes emancipados, con mayor autonomía, por tanto, de ingresos y mayores disposiciones para el empleo, parece llevar aparejado un abandono precoz de los estudios. Pero, al mismo tiempo, el proyecto migratorio de los jóvenes (a veces es un proyecto familiar) centrado en la búsqueda de empleo trae como consecuencia unas tasas de actividad de los jóvenes inmigrantes superiores a las de los españoles y, adicionalmente, menores posibilidades de elección (esto podría explicar la concentración en algunas ramas de actividad de peores condiciones laborales y salariales).

\section{Referencias bibliográficas}

Bourdieu, Pierre (1984). Questions de sociologie. París: Minuit.

CACHÓN, Lorenzo (2003a). "Desafíos de la "Juventud inmigrante" en la nueva "España inmigrante"». Revista de Estudios de Juventud, 60, marzo, 9-32.

- (2003b). Inmigrantes jóvenes en España: Sistema educativo y mercado de trabajo. Madrid: INJUVE.

CARRASCO, Concepción y RIESCO, Alberto (2008). «Escuela, consumo y mercado de trabajo: La producción de la juventud entre los jóvenes de origen inmigrante». Revista de Educación, 345, enero-abril, 183-203. Madrid: Ministerio de Educación y Ciencia. 
Colectivo Ióe (2002). Inmigración, escuela y mercado de trabajo: Una radiografía actualizada. Barcelona: Fundación La Caixa. Colección Estudios Sociales, 11.

CONSEJO ECONÓMICO Y SOCIAL (2006). Mercado de trabajo, políticas de empleo y relaciones laborales. Madrid.

García Borrego, Iñaki (2003). «Los hijos de inmigrantes como objeto de estudio de la sociología». Anduli: Revista Andaluza de Ciencias Sociales, 3, 27-46.

InSTITUTO NACIONAL DE ESTADÍSTICA (2006). Encuesta de estructura salarial. Madrid. http://www.ine.es/inebase/cgi/um? $\mathrm{M}=\% 2 \mathrm{Ft} 22 \% 2 \mathrm{Fp} 133 \& \mathrm{O}=$ inebase $\& \mathrm{~N}=\& \mathrm{~L}=$

- (2007). "Ficheros de microdatos». Encuesta de población activa. Madrid.

- (2008). Padrón municipal: explotación estadística, 1 de enero. http://www.ine.es/inebase/cgi/um? $\mathrm{M}=\% 2 \mathrm{Ft} 20 \% 2 \mathrm{Fe} 245 \& \mathrm{O}=$ inebase $\& \mathrm{~N}=\& \mathrm{~L}=$

López Blasco, Andreu; Cachón, Lorenzo; Comas, Domingo; Andreu, Jaime; Aguinaga, Josune y NAVARRETE, Lorenzo (2005). Informe Juventud en España 2004: Condiciones de vida y situación de los jóvenes. Madrid: Instituto de la Juventud.

Martín CRIAdo, Enrique (1998). Producir la juventud: Crítica de la sociología de la juventud. Madrid: Istmo.

MiNisTERIO DE EDUCACIÓN Y CIENCIA (2005). Encuesta de transición educativo-formativa e inserción laboral. Madrid._http://www.mec.es/mecd/jsp/plantilla.jsp?id= 33\&area=estadisticas

Ministerio de Trabajo e Inmigración (2007). Anuario de estadísticas. Madrid. http://www.mtas.es/estadisticas/anuario.htm

ObSERVATORIO PERMANENTE DE LA INMIGRACIÓN (2006). Informe estadístico. Madrid: Ministerio de Trabajo y Asuntos Sociales. http://inmigrantes.mtas.es/es/general/DatosEstadisticos_index.html

Piore, Michael (1983). «Los trabajadores inmigrantes». En: Piore, M. (comp.). Paro e inflación: Perspectivas institucionales y estructurales. Madrid: Alianza, 273-289. 